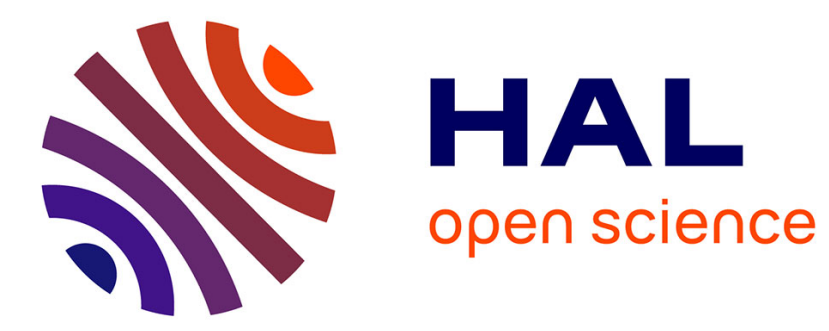

\title{
De la pornographie illégale aux sex-shops
}

Baptiste Coulmont

\section{To cite this version:}

Baptiste Coulmont. De la pornographie illégale aux sex-shops. Annales de Dermatologie et de Vénéréologie, 2012, 139, pp.859-861. 10.1016/j.annder.2012.09.004 . halshs-00762935

\section{HAL Id: halshs-00762935 https://shs.hal.science/halshs-00762935}

Submitted on 9 Dec 2012

HAL is a multi-disciplinary open access archive for the deposit and dissemination of scientific research documents, whether they are published or not. The documents may come from teaching and research institutions in France or abroad, or from public or private research centers.
L'archive ouverte pluridisciplinaire HAL, est destinée au dépôt et à la diffusion de documents scientifiques de niveau recherche, publiés ou non, émanant des établissements d'enseignement et de recherche français ou étrangers, des laboratoires publics ou privés. 


\section{De la pornographie illégale aux sex-shops}

English : From illegal pornography to sex stores

\section{Baptiste Coulmont}

Préprint non corrigé d'un article paru dans Annales de dermatologie et de vénéréologie http://dx.doi.org/10.1016/j.annder.2012.09.004

Mots-clefs : pornographie, commerce, droit, histoire, sociologie

Keywords : pornography, commerce, law, history, sociology

Quel était l'état du commerce pornographique avant l'arrivée des sex-shops, et comment ces magasins en sont venus à endosser l'image qu'ils ont aujourd'hui.

\section{L'épuisement d'un concept, « l'outrage aux bonnes mœurs »}

Jusque vers 1973-1974, c'est la notion juridique d' « outrage aux bonnes mœurs » qui permet de réguler le commerce de la pornographie (sous forme d'images, films, ou d'objets comme les godemichets, et les vibromasseurs...). Qu'est-ce que cet « outrage aux bonnes mœurs »? C'est l'article 283 du Code pénal : «Sera puni d'un emprisonnement d'un mois à deux ans et d'une amende de $360 \mathrm{~F}$ à $30.000 \mathrm{~F}$ quiconque aura : Fabriqué ou détenu en vue d'en faire commerce, distribution, location, affichage ou exposition ; Importé ou fait importer, exporté ou fait exporter, transporté ou fait transporter sciemment aux mêmes fins ; Affiché, exposé ou 
projeté aux regards du public ; Vendu, loué, mis en vente ou en location, même non publiquement ; Offert, même à titre gratuit, même non publiquement, sous quelque forme que ce soit, directement ou par un moyen détourné ; Distribué ou remis en vue de leur distribution par un moyen quelconque, Tous imprimés, tous écrits, dessins, affiches, gravures, peintures, photographies, films ou clichés, matrices ou reproductions phonographiques, emblèmes, tous objets ou images contraires aux bonnes mœurs. »

Mais l'« outrage aux bonnes mœurs » (OBM dans le langage policier) est, dans les années soixante-dix, un concept juridique en crise (1). Les juges arrêtent progressivement de condamner pour outrage aux bonnes mœurs : 351 personnes sont condamnées en 1962, 220 dix ans plus tard, et une vingtaine en 1984. L'épuisement de la notion est perceptible dès le tournant des années soixante. En 1969, il y a moins d'une centaine de condamnations, quand Jacques Chaban-Delmas, premier ministre, déclare nécessaire l'entrée dans une « nouvelle société », définie comme : « une société prospère, jeune, généreuse et libérée ».

Au même moment, entre 1967 et 1974, le commerce parisien de la pornographie se modifie fortement. C'est une période mouvementée pendant laquelle le monde des petits dealers de la place Pigalle et du boulevard de Clichy s'efface, un monde dans lequel la vente par correspondance semble exploser, un monde qui voit l'ouverture d'une légion de boutiques revendiquant publiquement l'enseigne de «sex-shop ». Il va falloir réguler cette nouvelle organisation commerciale.

Avant 1970, les policiers concentrent leur action sur des vendeurs indépendants, des commerces clandestins. Le repérage du commerce se fait grâce à la surveillance quotidienne de quelques lieux (principalement Pigalle) et grâce au travail d'informateurs. La police est prévenue du commerce illicite par les concurrents des petits dealers et par des indicateurs... 
Les policiers saisissent nombre d'objets artisanaux, des objets bricolés, fabriqués dans un but personnel ou pour une circulation limitée. Les vibromasseurs, les godemichets... sont parfois des productions individuelles.

\section{Des magasins particuliers}

À partir de 1970 apparaissent, à Paris, des magasins spécifiquement orientés vers la vente d'objets érotiques : livres, disques, gadgets, posters, photographies, films parfois... (2)

Ces magasins apparaissent donc rapidement comme des magasins un peu particuliers. Les « sex-shops » sont très rapidement perçus comme quelque chose de neuf.

Des médiateurs culturels s'emparent du terme de sex-shop. En 1970-1971, une série de dessins humoristiques va diffuser l'image de ce nouveau commerce. Les dessinateurs d'humour utilisent le cadre des sex-shops pour montrer la rapide évolution des mœurs.

Les sex-shops concentrent en un lieu toute une série d'objets érotiques... et c'est sur ce point que la police va agir. La proximité physique de certains objets entre eux, plus que les objets en eux-même, va être codée par les policiers comme un motif d'action. Avant 1970, c'était l'objet en lui-même qui était repéré (ainsi que le lieu de vente, le type de vendeur...). Avec l'arrivée des sex-shops, c'est la réunion, en un même lieu, d'objets qui, séparément, ne sont pas outrageants pour les bonnes mœurs, qui devient une des bases de l'action.

Les « dossiers de procédure » conservés aux Archives de Paris (et générés suite aux interpellations pour « outrage aux bonnes mœurs ») nous apprennent, en creux, beaucoup sur 
le caractère mouvant des décisions policières (3). Une statue-moulage, qui n'avait pas été jugée problématique lors d'une précédente visite des policiers, peut devenir quelques mois plus tards, l'objet central déclenchant une inculpation pour outrage aux bonnes mœurs une fois que la situation locale, rue Saint-Denis, demande l'intervention des policiers.

Les juges se rendent compte que les objets saisis : cache-sexe tricotés, vibromasseurs, statuettes, bougies... ne sont pas, en eux-même outrageants pour les bonnes mœurs. Plusieurs jugements vont préciser que c'est bien la réunion, en un même endroit, de ces objets, qui provoque les sens, excite l'imagination et les plus bas instincts de l'homme. L'outrage aux bonnes mœurs n'est pas substantif, il est relationnel.

Ainsi le moulage en plastique, taille réelle, d'une jeune femme nue se caressant l'entrejambe... est jugé outrageant dans un sex-shop, mais pas dans une galerie d'art, ce que souligne l'artiste qui a réalisé le moulage. «Je ne pense pas que le moulage de cette femme, pris séparément puisse avoir un caractère pornographique. Seule son exposition au milieu d'autres objets qui ont, eux, un caractère pornographique pourrait choquer certaines personnes. » (Archives de Paris, 1914W 23)

Les juges semblent partiellement d'accord avec cette interprétation : « il appartient au Tribunal de rechercher ce caractère [outrageant] soit à raison des particularités intrinsèques des objets, soit de leur mode de présentation et de l'intention d'éveiller la curiosité ou les instincts malsains de la clientèle à lequelle ils sont offerts. » (Archives de Paris, 2211W 260, Tribunal correctionnel de Paris, 17e chambre, Audience du 2 avril 1971) Les vibromasseurs sont vendables. Les préservatifs (fantaisie ou non) le sont aussi. Mais réunis, ils constituent un outrage aux bonnes mœurs : « l'appareil dit “vibro masseur” ne saurait être en lui-même considéré comme outrageant pour les bonnes mœurs, mais [...] décoré d'un préservatif fantaisie, il attire l'attention sur son utilisation sexuelle et entre dès 
lors dans le champ d'application de la loi pénale » (Archives de Paris, 2211W 260, Tribunal correctionnel de Paris, 17e chambre, Audience du 2 avril 1971)

Diverses affaires portent donc sur des objets, et une jurisprudence mouvante se met en place concernant poupées gonflables, vibromasseurs et autres godemichets... Cette évolution des positions des juges échappent en partie aux juristes, qui ne se sont pas penchés, dans les revues de droit, sur ces sujets scabreux et sans doute un peu ridicules.

Le droit, toutefois, n’est pas seulement « répressif ». Il est aussi « producteur », au sens où des stratégies commerciales ont pu s'appuyer sur lui (4). On trouve par exemple, annexée à une procédure pour OBM, des dépliants publicitaires pour les « Productions du dragon », une librairie par correspondance vendant aussi préservatifs, vibromasseurs et une « vibrocrème spéciale », « pour une parfaite utilisation du vibromasseur ». Dans ce dépliant, voici comment certains ouvrages sont présentés : «Par décrets du ministère de l'intérieur, publiés au journal officiel de la République française, la publicité et la vente même de l'un ou l'autre de ces titres peuvent se trouver interdites du jour au lendemain. Nous sommes donc obligés de les retirer immédiatement de notre liste. Si donc un titre vous intéresse parmi ceux indiqués ciaprès, il est de votre intérêt de le commander le plus rapidement possible... » (Archives de Paris, 1914W 51)

La répression, l'interdiction, la menace juridique, sont ici utilisées comme incitation à l'achat. Dans le même ordre d'idées, il semble que le but des juges et des policiers ne fut pas de faire disparaitre les sex-shops, mais de forger, par le droit, la possibilité de réguler ces nouveaux commerces. Même si ces magasins ne vendent que des objets qui, en eux-mêmes, sont autorisés... leur réunion dans un but commercial leur donne un caractère outrageant (un peu comme les lettres E, S et X qui ne posent pas problème... mais qui, une fois composées, forment un mot, SEXE, qui peut poser problème). 
Cette conception de l'outrage aux bonnes mœurs ne perdure pas : rapidement les juges conviennent que le sex-shop peut même contenir des objets outrageants... si l'entrée du magasin est réservée aux personnes averties.

\section{Créer une classe particulière de magasins}

Très vite avec l'apparition de magasins portant, en enseigne, les mots « sex-shop », le préfet de police semble se rendre compte qu'il va falloir organiser leur existence en plus de chercher à limiter leur nombre. L'outrage aux bonnes mœurs étant en voie d'épuisement, le préfet choisit le mode d'action suivant : il va considérer ces magasins comme des librairies vendant principalement des publications interdites aux mineurs. Il va demander que, puisque les mineurs ne peuvent acheter, il faut leur interdire l'entrée (ordonnance n70-15878).

Au lieu de mettre au centre de son action la morale sociale, les « bonnes mœurs », le préfet met au centre de son action une catégorie spécifique, les enfants (plus précisément les mineurs de 18 ans). Ce n'est plus la morale de la société que remettent en cause les sex shops, c'est la santé morale des mineurs. Une classe de magasins est définie, qui fait l'objet d'une surveillance spécifique, et de règles de fonctionnement particulières. Mais séparer les « sexshops » des autres magasins c'est aussi les distinguer des autres magasins, les rendre encore plus visibles sur le tissu urbain.

A partir de 1974-1975, les sex-shops deviennent des lieux destinés en partie à la masturbation masculine. Des cabines individuelles proposant le visionage de films pornographiques font 
leur apparition. Et après la loi créant la catégorie des films X, le nombre de cinémas proposant des films pornographique diminue fortement : les sex-shops récupèrent ce commerce.

En conséquence leur image change fortement. Ce ne sont plus des lieux mixtes, les vendeuses disparaissent au profits de vendeurs chargés de contrôler les espaces destinés à la masturbation, les magasins restent ouvert tard la nuit, ils apparaissent comme des lieux destinés à répondre à la « misère sexuelle » (une expression que l'on ne voit pas utilisée avant le milieu des années 1970).

\section{Les oppositions violentes}

Des séries d'actes violents dirigés contre les sex-shops se remarquent assez vite à la lecture de la presse de l'époque. Au début des années 1970, ils semblent être des actes individuels ou déclenchés par colère : bris de vitrines à Paris, incendie d'un magasin à Lyon.

Le $1^{\text {er }}$ juin 1971, le quotidien Lyon Matin raconte ainsi la destruction d'un sex-shop local : «Une seule vitrine devait être épargnée par les flammes. À l'intérieur de celle-ci, le public povait lire une lettre manuscrite anonyme rédigée en ces termes : "Etant décidé, à nettoyer Lyon, un jour que votre salle boîte sera pleine ; la nuit nous irons avec des bidons d'essence pour brûler tout ce fumier comme à Saint-Laurent du Pont. Avis.” Le magasin ouvert il y a plusieurs mois avait suscité critique et controverses. Le 8 novembre 1970, nos services recevaient une lettre à l'intitulé suivant : “Tout le quartier des Terreaux en ebulition (sic) dit non à la pornographie" "

En 1975, toujours à Lyon, les actions contre les sex-shops proviennent d'un autre secteur de la société, les prostituées, qui protestent, de manière organisée, contre la pornographie légale : 
« Dénonçant la pornographie autorisée, n’acceptant pas la liberté d'expression de ces cinémas relégués à ces productions bien spéciales, ces dames se sont acharnées l'autre nuit, le pinceau à la main. Une dizaine de façades ont été badigeonnées ainsi que deux « Sex-shops » du même quartier. Le Tout-Lyon vacancier a pu observer aux premières heures de la matinée les chefs-d'œuvre des péripatéticiennes. Bleu, rouge, vert, noir, toutes les couleurs étaient de la fête et ornaient les vitrines d'exposition et hall d'entrée des cinémas qui projettent des films érotiques. De larges inscriptions expliquaient en outre, ce mouvement spontané et diversement accueilli. “Qui débauche nos enfants ? Eux ou nous ?” » (Dernière heure lyonnaise, 23 août 1975) (5).

En 1976 à Paris un « groupe croisé » revendique l'attaque de trois sex-shops, par un appel à l'AFP justifiant ces actes par la « guerre contre la dégradation morale de la France par la pornographie » (on ne trouve trace de cette attaque que dans le Washington Post du 22 février 1976, p.B2). En 1976 toujours, à Nantes, en marge du procès de quelques éducateurs ayant fourni des préservatifs à des adolescents, une manifestation procède à la mise à sac de deux magasins : la revue d'éducation spécialisée Champ social en rendra compte dans un numéro spécial (6).

En 1978, enfin, c'est lors des manifestations de la journée des femmes (au début du mois de mars), que des magasins sont attaqués, de manière quasi systématique, dans plusieurs villes de France. Une féministe française, Françoise d'Eaubonne, qui proteste en 1971 contre les fermetures administratives de certains sex-shops, écrit en 1978 que le plasticage de certains sex-shops est « une entreprise considérablement plus saine que celle des militantes du programme commun des femmes » qui réclament une commission de censure (7).

Certes, les années soixante-dix étaient des années de plus grande violence urbaine « radicale », mais ces séries d'attaques, en provenance aussi bien de l'extrême-gauche féministe que de l'extrême-droite croisée, de travailleuses du sexe qui revendiquent un statut 
de profession libérale que d'éducateurs sociaux syndiqués à la CFDT... ces séries d'attaques donc pointent la place impossible du petit commerce du sexe après la libéralisation morale giscardienne. Les libéralismes moraux et économiques sont en conflit. Les sex-shops apparaissaient vers 1969-1970 comme des antennes commerciales de la libération sexuelle, ils apparaissent désormais comme des profiteurs de la misère sexuelle, comme des exploiteurs sexistes.

\section{Références}

1. Lochak, D. (1994) Le droit à l'épreuve des bonnes mœurs. Dans Les bonnes mœurs, pp 1553, Presses Universitaires de France, Paris.

2. Coulmont, B. (2007) Sex-shops, une histoire française, Editions Dilecta, Paris.

3. Coulmont, B. (2009) L'Affaire Olesniak, Genre, sexualité, société 2.

4. Foucault, M. (1976) Histoire de la sexualité. La volonté de savoir, Gallimard, Paris.

5. Mathieu, L. (2001) Mobilisations de prostituées, Belin, Paris.

6. (1976) Le procès des éducateurs, Champ social 20, 18-20.

7. Eaubonne (d'), F. (1978) Contre violence ou la Résistance à l'État, Tierce, Paris.

\section{Coordonnées :}

Baptiste Coulmont, maître de conférences, sociologie, Université Paris 8, Laboratoire CRESPPA, 59-61 rue Pouchet, 75017 Paris, 